\title{
DEVELOPMENT OF A MULTI-MEGAWATT CIRCULATOR FOR X-BAND
}

\author{
Jeff Neilson and Lawrence Ives, Calabazas Creek Research, Inc., Saratoga, CA 95070 \\ Sami G. Tantawi, Stanford Linear Accelerator Center, Stanford, CA 94309
}

Research is in progress on a $\mathrm{TeV}$-scale linear collider that will operate at 5-10 times the energy of presentgeneration accelerators. This will require development of high power RF sources generating of 50-100 MW per source. Transmission of power at this level requires overmoded waveguide to avoid breakdown. In particular, the $\mathrm{TE}_{01}$ circular waveguide mode is currently the mode of choice for waveguide transmission at Stanford Linear Accelerator Center (SLAC) in the Multimode Delay Line Distribution System (MDLDS).

A common device for protecting an RF source from reflected power is the waveguide circulator. A circulator is typically a three-port device that allows low loss power transmission from the source to the load, but diverts power coming from the load (reflected power) to a third terminated port. To achieve a low loss, matched, three port junction requires nonreciprocal behavior. This is achieved using ferrites in a static magnetic field which introduces a propagation constant dependent on RF field direction relative to the static magnetic field.

Circulators are currently available at X-Band for power levels up to $1 \mathrm{MW}$ in fundamental rectangular waveguide; however, the next generation of RF sources for $\mathrm{TeV}$-level accelerators will require circulators in the 50-100 MW range. Clearly, conventional technology is not capable of reaching the power level required.

In this paper, we discuss the development of an XBand circulator operating at multi-megawatt power levels in overmoded waveguide. The circulator will employ an innovative coaxial geometry using the $\mathrm{TE}_{01}$ mode. Difficulties in maintaining mode purity in oversized rectangular guide preclude increasing guide area to allow increasing the power level to the desired 50-100 MW range.

The $\mathrm{TE}_{01}$ mode in circular waveguide is very robust mode for transmission of high power in overmoded waveguide $^{2}$. The mode is ideal for transmission of high power microwaves because of its low-losses, zero tangential field on the guide (which minimizes arcing problems) and reduced propensity for mode conversion compared to non-asymmetric circular waveguide modes. Unfortunately, no current designs exist for circulators using the circular $\mathrm{TE}_{01}$ mode.

The basic building block for all low-loss circulators and isolators is a nonreciprocal element with a phase shift dependent on the propagation direction in the guide. Such an element can be constructed by placement of a hollow ferrite rod in a cylindrical waveguide. An inner conductor placed inside the ferrite rod conducts a current pulse that induces an azimuthal magnetic field inside the ferrite. This configuration is depicted in Figure 1a. An alternate configuration using permanent magnets is shown in (a)

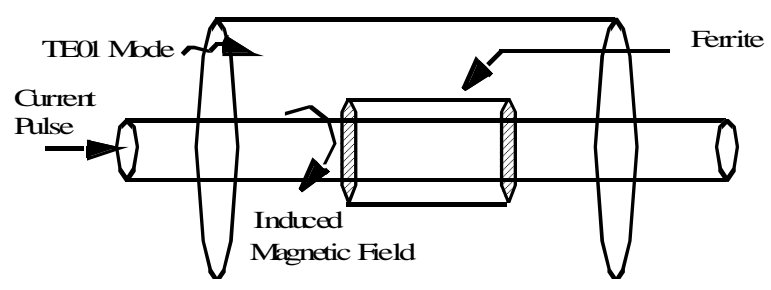

(b)

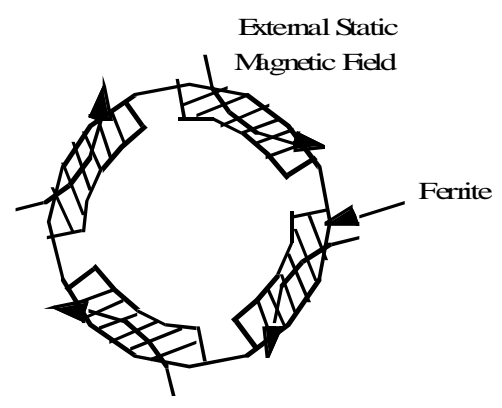

Figure 1. Configurations for nonreciprocal element for TE01 mode in circular waveguide. (a) Inner cylindrical placement of ferrite with azimuthal field induced by current pulse through conductor inside ferrite. (b) Transverse cross section of cylindrical guide with ferrites on outer radius guide with azimuthal field induced by external permanent magnet field.

Figure 1b. Either of these configurations will create a different phase shift for waves propagating in opposite directions along the waveguide axis. This feature can be used to develop a high power circulator.

We are currently testing a $\mathrm{TE}_{01}$ nonreciprocal phase shifter in a $50 \mathrm{MW}$ test stand. This device is in the configuration shown in Figure 1a. The induced differential phase shift and loss will be measured and compared to calculations.

This work was supported by U.S. DOE Small Business Innovative Research grant DOE DE-FG03$01 \mathrm{ER} 83210$.

1. P.B. Wilson, "RF power sources for $5-15 \mathrm{TeV}$ linear colliders," Intern. workshop on pulsed RF sources for linear colliders, Shonan Village, Japan, April 1996.

2. Theodore Moreno, Microwave Transmission Design Data, Dover Publications, New Your, NY, 1948. 